\title{
STELLAR MODELS WITH CONVECTION AND WITH DISCONTINUITY OF THE MEAN MOLECULAR WEIGHT
}

\author{
P. LEDOUX \\ Yerkes Observatory \\ Received December 9, 1946
}

ABSTRACT

In this paper the effect of a discontinuity in the mean molecular weight, $\mu$, in stellar models is examined. The case when such a discontinuity occurs in the envelope $\left(r / R<\frac{1}{2}\right.$ and $\left.m[r] \simeq M\right)$ is first considered. And it is shown that, in general, a discontinuity in $\mu$ produces convective instability in a small zone past the place where the discontinuity occurs. The resulting turbulence will cause mixing, and the star will rapidly adjust itself to a neighboring stable state, in which the interior region of higher $\mu$ and the exterior region of lower $\mu$ are separated by a transition region in which $\mu$ varies according to the law $\mu \propto P^{7 / 5}$. It is further shown that the time required for such a readjustment is very small, compared to the time in which a discontinuity in $\mu$ can be established.

The case in which the discontinuity in $\mu$ occurs in the deep interior is next examined. It appears that, even here, a pure discontinuity of $\mu$ will, in general, be smoothed out and a transition zone of variable $\mu$ established. The law of variation of $\mu$ in this transition zone follows the law $\mu \propto m(r) P^{7 / 5}$. Owing to the presence of the factor $m(r)$, the importance of the transition zone is greater when the change in $\mu$ occurs in the deep interior.

Finally, stellar models are constructed which consist of convective cores and radiative envelopes with assigned mean molecular weights $\mu_{i}$ and $\mu_{e}$, respectively, separated by transition zones of variable $\mu$ (also in radiative equilibrium). It is shown that these models satisfy all the conditions of the problem and, further, that they do not differ greatly in their physical properties from models constructed with point-source envelopes fitted directly to convective cores without regard to the continuity of the luminosity at the interface. However, up to a certain point their interpretation as a sequence of evolution is easier.

1. Introduction.-Stellar models in which the mean molecular weight, $\mu$, takes different values in different parts of the star have been discussed on a number of occasions in recent years. The most important physical context in which the problem arises is in connection with the expected increase in the mean molecular weight in the convective regions in the interior, consequent to the gradual impoverishment of hydrogen by the continued operation of the carbon cycle. ${ }^{1}$ F. Hoyle and R. A. Lyttleton ${ }^{2}$ have also considered the possibility that accretion of interstellar hydrogen might lead to a discontinuity of $\mu$ in the outer parts of a star also. Particularly in this latter case, these authors conclude from a discussion of the conditions to be satisfied at an interface, where $\mu$ jumps discontinuously from a value $\mu_{e}$ in the outer part to a value $\mu_{i}$ in the interior, that the ratio $\mu_{i} / \mu_{e}$ cannot exceed a certain limiting value $(\simeq 1.53)$; and they further infer from this that when $\mu_{i} / \mu_{e}$ exceeds this critical value, developments of a dynamical nature must take place, with large-scale currents setting in; they even envisage the possibility of nonstatic atmospheres arising in this way. However, a detailed examination of this problem undertaken in $\S \S 3-5$ reveals that these conclusions are without any real foundations.

Again it appears that there are also numerous misunderstandings regarding the problem considered by Schönberg and Chandrasekhar (op. cit). The discontinuity in $\mu$ assumed by these writers at the place $r_{c}$, where the radiative gradient becomes unstable, is a simplification of a mathematical nature which cannot, of course, be realized in practice. It is apparent that, even if a discontinuity should be artificially introduced at a given instant, we should expect that the "corners" will be smoothed by mixing at the

\footnotetext{
${ }^{1}$ M. Schönberg and S. Chandrasekhar, Ap. J., 96, 161, 1942; see also M. Harrison, Ap. J., 100, 343 $1944 ; 103,193,1945 ; 105,322,1947$.
}

${ }^{2} M . N ., 102,218,1942$. 
interface. Indeed, it might even be argued by some that large-scale currents of the nature expected in rotating stars will prevent any appreciable inhomogeneities from arising in the chemical composition of a star. However, we shall not consider this latter possibility and shall restrict ourselves to those processes of mixing which may be expected in a star in hydrostatic equilibrium. There are, then, two types of processes to be considered: mixing (or sedimentation) consequent to thermal or similar diffusion and mixing by turbulent eddies when these exist in virtue of a prevailing superadiabatic gradient. It would seem that the former, as an agency in bringing about or smoothing existing differences of chemical composition, is very inefficient. ${ }^{3}$ We are, therefore, left with mixing only in regions where convection exists or in regions where convection is set up in consequence of a difference of $\mu$ in neighboring regions; in the latter case, we may expect the mixing to cease, once an appropriate redistribution has been effected. And, since turbulent eddies are known from meteorological experience to be very efficient as an agency for mixing, we shall restrict ourselves to this mechanism alone in our further considerations. One consequence which follows on these assumptions is that $\mu$ will have a constant value in the (Cowling) convective core and that the variation of $\mu$ outside this region must be steep. This is the justification for the assumption of a discontinuous $\mu$ made in the investigation we have referred to. However, we shall show in this paper how it is possible to avoid the strictly nonphysical assumption of a mathematical discontinuity in $\mu$ by deriving the variation of $\mu$ which will result in the outer radiative regions in consequence of turbulence having once existed.

2. Preliminary considerations. - It is well known that, for stars of normal masses, radiation pressure as a factor in the equation of hydrostatic equilibrium can be ignored, and the equations of the problem are

$$
\begin{aligned}
& \frac{d P}{d r}=-\frac{G m(r) \rho}{r^{2}} \\
& \frac{d T}{d r}=-\frac{3 \kappa \rho}{16 \pi a c T^{3}} \frac{L(r)}{r^{2}}
\end{aligned}
$$

or

$$
\frac{d \log T}{d \log P}=\frac{3 \kappa_{0} \mu\left(1-X^{2}\right)}{16 \pi a c G \Re} \frac{L(r)}{m(r)} \frac{P^{2}}{T^{8.5}},
$$

where we have used Kramer's law of opacity,

$$
\kappa=\kappa_{0}\left(1-X^{2}\right) \rho T^{-3.5} .
$$

In the foregoing equations, $X$ is the abundance of hydrogen. To the same order of approximation, the mean molecular weight is given by

$$
\mu=\frac{2}{1+3 X} \text {. }
$$

Further, we have

$$
P=\frac{\Re \rho T}{\mu}
$$

and

$$
d L(r)=4 \pi \rho \epsilon r^{2} d r
$$

where $\epsilon$ is the rate of generation of energy and

$$
d m(r)=4 \pi \rho r^{2} d r
$$

${ }^{3}$ S. Chapman, M.N., 82, 292, 1922. 
In a region where $\mu$ is a constant, the condition of convective stability resulting from the requirement that a small parcel of matter displaced adiabatically upward (downward) becomes heavier (lighter) than the surrounding medium is

$$
\frac{d \log T}{d \log P} \leqslant \frac{2}{5}
$$

if the ratio of specific heats, $\gamma$, is taken to be $\frac{5}{3}$, as for a monatomic gas.

In a region where $\mu$ varies (owing to a real spatial difference in chemical composition and not to the variation of physical conditions), we must take into account the fact that $\mu$ remains constant during the displacement of a small element, and the condition of stability becomes

$$
\frac{d \log T}{d \log P} \leqslant \frac{2}{5}+\frac{d \log \mu}{d \log P}
$$

Now, if $\mu$ varies from a value $\mu_{e}$ in the outer part to a value $\mu_{i}=\mu_{e}+\Delta \mu$ in the inner part across a spherical shell of thickness $\Delta r$ between the two parts, then in this region of varying $\mu$, equations (1)-(7) will continue to be valid, whatever the value of the ratio $\Delta \mu / \Delta r$. Equations (1) and (7) require that $\Delta m(r)$ and $\Delta P$ be proportional to $\Delta r$, while from equation (5) it follows that one of the ratios $\Delta \rho / \Delta r$ or $\Delta T / \Delta r$ must be of the same order of magnitude as $\Delta \mu / \Delta r$. L. Gratton ${ }^{4}$ argues in favor of taking $\Delta T / \Delta r$ proportional to $\Delta \mu / \Delta r$. But, by equation (2), this would make $L(r)$ depend, in that region, on the steepness of the variation of $\mu$, which is physically inadmissible; and $\Delta T$, like $\Delta m(r)$ and $\Delta P$, should be proportional to $\Delta r$. Passing to the limit of a discontinuity in $\mu$, we obtain the usual boundary conditions, namely, that $m(r), P$, and $T$ are continuous across the interface, while $\rho$ has a discontinuity related to that of $\mu$ by

$$
\frac{\rho_{i}}{\rho_{e}}=\frac{\mu_{i}}{\mu_{e}}
$$

These are the boundary conditions used by Schönberg and others in their investigations.

As $\Delta T$ and $\Delta P$ are proportional to $\Delta r$, while $\Delta \mu$ is independent of it, condition (9) shows that such a region of variation of $\mu$ will become extremely stable with respect to convection as $\Delta r$ decreases.

By equation (6) $L(r)$ also should be continuous across the interface. It is this condition that Hoyle and Lyttleton ${ }^{2}$ have taken explicitly into account in their investigation of the external layers of a star. Recently, ${ }^{4}$ they have extended their discussion to the deep interior, considering especially the case in which the discontinuity of $\mu$ occurs at the boundary of the convective core. They consider that, since, by definition, convection should stop at the boundary of the core, the transfer of energy on both sides of the interface should be governed by equation (2), and the continuity of $L(\boldsymbol{r})$ at the interface would require, according to equation (3), that

$$
\left(n_{e}+1\right) \mu_{e}\left(1-X_{e}^{2}\right)=\left(n_{i}+1\right) \mu_{i}\left(1-X_{i}^{2}\right),
$$

where we have introduced the effective polytropic index $n$,

$$
(n+1)=\frac{d \log P}{d \log T}
$$

${ }^{4}$ Dr. Gratton's original paper is not accessible to the author, but, through the kindness of Dr. Chantrasekhar, the author has seen some correspondence in which Dr. Gratton has stated his argument. Similarly, the author has also had the opportunity of seeing a manuscript of a paper by Hoyle and Lyttleton on the same subject. 
Hoyle and Lyttleton point out that condition (11) is incompatible with the procedure generally adopted in fixing the boundary of the convective core $\left(n_{i}=\frac{8}{2}\right)$ where the radiative solution for the point-source envelope becomes unstable $\left(n_{e}=\frac{3}{2}\right)$, except in the case in which there is no change of composition across the interface $\left(\mu_{i}=\mu_{e}\right)$. Furthermore, condition (11) implies a limitation for the possible variation of $\mu$, just as in the external layers. Thus, when $\mu_{i}$ is different from $\mu_{e}$, Hoyle and Lyttleton propose to replace the condition of the continuity of $n$ across the interface by condition (11), whenever it can be satisfied. However, we shall show $(\$ \$ 5,6)$. that condition $(11)$, even when it is satisfied for $n_{i}=\frac{3}{2}$, does not, in itself, tell us anything as to the existence or otherwise of a convective region past the interface. Also, the limit formally set by equation (11) on the magnitude of the variation of $\mu$ can be violated in the interior of the star just as in the external layers without endangering its equilibrium. In fact, we shall show $(\$ \S 5,6)$, contrary to the views expressed by Hoyle and Lyttleton, that for any assigned value of $\mu_{i} / \mu_{e}$ equilibrium configurations can be found which will be compatible with all the conditions of the problem, including the condition of the continuity of $L(r)$ and the stability criteria (8) and (9). But it may be said, even here, that these detailed considerations confirm what may indeed be expected, on general grounds - that a satisfactory first approximation is obtained by insuring the continuity of $n$ across the interface and ignoring condition (11). Thus a stellar model, constructed with the usual boundary conditions of continuity of $n$ and discontinuity of $\rho$ at the interface, where $\mu$ is discontinuous, will violate condition (11). The consequence of this violation is that more energy is leaving the external side of the interface than is brought to its internal side by radiation alone. But it is well known that, in the convective parts of the star, turbulence is very effective as a means for transporting energy ${ }^{5}$ so that only a very slight superadiabatic gradient extending to the surface of the convective core would be sufficient to compensate for this. Thus, if we suppose, for example, that the two kinds of material composing the external and the internal regions cannot mix at all (or that they are separated by a perfectly conducting membrane of negligible mass), a finite pressure, $p_{c}$, due to turbulence will be applied to the base of the external part, and it should be taken into account in the equations of fit. However, just like the very slight degree of superadiabasy necessary, this pressure will be very small compared to $P$, so that the external solution to be chosen will not be very different from the one corresponding to the ordinary fitting or, on the other picture, the membrane between the two parts need sustain only very small stresses.

In reality, some mixing will take place at the interface, and we shall see how it leads to a stable state free of any difficulties arising from condition (11).

3. The solution for a discontinuity of $\mu$ in the external layers. - In the external layers of a star, $M$ and $L$ can be considered as constants, and equations (1) and (3) reduce to

and

$$
\frac{d P}{d r}=-\frac{G M}{r^{2}} \rho
$$

$\frac{1}{T} \frac{d T}{d r}=C\left(1-X^{2}\right) \mu \frac{P^{2}}{T^{8.5}} \frac{1}{P} \frac{d P}{d r}$,

where

$$
C=\frac{3 \kappa_{0}}{16 \pi a c G \Re} \frac{L}{M} .
$$

If we use equation (14), the conditions of stability (8) and (9) become, respectively,

and

$$
C\left(1-X^{2}\right) \mu \frac{P^{2}}{T^{8.5}} \leqslant \frac{2}{5}
$$

$$
C\left(1-X^{2}\right) \mu \frac{P^{2}}{T^{8.5}}-\frac{d \log \mu}{d \log P} \leqslant \frac{2}{5} .
$$

${ }^{5}$ Cf., e.g., Cowling, M.N., 96, 51-52, 1935. 
First, we shall assume that a discontinuity of $\mu$ occurs at $r=r_{c}$ (say).

The solution for $T$ in the external part $\left(r_{c} \leqslant r \leqslant R\right)$ is well known ${ }^{6}$ and can be written in the form

$$
T_{e}=\frac{G M \mu_{e}}{4.25 \Re}\left[\frac{1}{r}-\frac{1}{R}\right] .
$$

The other variables are related to $T$ by

or

$$
C\left(1-X_{e}^{2}\right) \mu_{e} P_{e}^{2}=\frac{1}{4.25} T_{e}^{8.5}
$$

$$
\frac{P_{e}}{P_{e c}}=\left(\frac{T_{e}}{T_{e c}}\right)^{4.25} \quad \text { and } \quad \frac{\rho_{e}}{\rho_{e c}}=\left(\frac{T_{e}}{T_{e c}}\right)^{3.25} \text {. }
$$

Obviously, condition (16) is satisfied, and the radiative equilibrium is stable in that region.

In the inner part, close enough to the interface, $L$ and $M$ can still be considered as constant, and the solution can still be obtained by integrating equation (14). After determining the constant of integration by the continuity of $P$ and $T$ at the interface, which requires that $P_{i c}$ and $T_{i c}$ satisfy equation (19), we have

$$
C\left(1-X_{i}^{2}\right) \mu_{i} \frac{P_{i}^{2}}{2}=\frac{T_{i}^{8.5}}{8.5}\left[1-\left(\frac{T_{i c}}{T_{i}}\right)^{8.5}\left(1-\frac{1-X_{i}^{2}}{1-X_{e}^{2}} \frac{\mu_{i}}{\mu_{e}}\right)\right] .
$$

Introducing the value of $P_{i}^{2} / T_{i}^{8.5}$ from equation (21) in equation (14) and replacing $d P / d r$ by its value (13), we obtain

$$
\frac{d T_{i}}{d r}=-\frac{1}{4.25} \frac{G M \mu_{i}}{\Re r^{2}}\left[1-\left(\frac{T_{i c}}{T_{i}}\right)^{8.5}\left(1-\frac{\left(1-X_{i}^{2}\right) \mu_{i}}{\left(1-X_{e}^{2}\right) \mu_{e}}\right)\right] .
$$

When we write

$y=\frac{T_{i}}{T_{i c}}, \quad r=\frac{1}{x}, \quad a=\frac{1}{4.25} \frac{G M \mu_{i}}{\Re T_{i c}}=\frac{\mu_{i}}{\mu_{e}} \frac{R r_{c}}{R-r_{c}}, \quad \beta=-\left(1-\frac{1-X_{i}^{2}}{1-X_{e}^{2}} \frac{\mu_{i}}{\mu_{e}}\right)$,

equation (22) becomes

$$
\frac{d y}{d x}=\alpha\left(1+\frac{\beta}{y^{8.5}}\right) .
$$

To obtain the exact solution of equation (23) is rather cumbersome, but, as we shall need it only in the immediate vicinity of the interface, where $y$ is very close to 1 , we can use a suitable approximation. Thus, if we wish to evaluate $y$ as a function of $x$, we can integrate equation (23) by the method of successive approximations. In the second approximation we find

$$
\begin{aligned}
\frac{T_{i}}{T_{i c}}=1+\frac{\mu_{i}}{\mu_{e}} \frac{R r_{c}}{R-r_{c}} & \left(\frac{1}{r}-\frac{1}{r_{c}}\right) \\
& +\frac{\beta}{7.5(1+\beta)}\left\{1-\frac{1}{\left[(1+\beta) \frac{\mu_{i}}{\mu_{e}} \frac{R r_{c}}{R-r_{c}}\left(\frac{1}{r}-\frac{1}{r_{c}}\right)+1\right]}\right\} .
\end{aligned}
$$

On the other hand, if we wish to evaluate $x$ as a function of $y$, it is more convenient to write $y=1+z$ in equation (23); and, limiting ourselves to the second-order terms in the development of $(1+z)^{8.5}$, we find

$$
a d x=\frac{d z}{1+\beta\left[1-8.5 z+40.375 z^{2}\right]} .
$$

${ }^{6}$ Cf. S. Chandrasékhar, Introduction to the Study of Stellar Structure, p. 300. 
Finally, we may explicitly note that, in the solution of the problem that we have presented, $\rho$ has a discontinuity at $r=r_{c}$ in accordance with equation (10).

We have now all that is necessary to study the stability of the radiative solution in the neighborhood of the interface. Since we have integrated equations (13) and (14) exactly, the continuity of $L$ at the interface has, of course, been insured.

We have already seen that the radiative equilibrium is stable in the external part of the star. To investigate its stability in the internal part, near the interface, we introduce the value of $P_{i}^{2} / T_{i}^{8.5}$ given by equation (21), into condition (16), and we find

$$
\frac{1}{4.25}\left[1-\left(\frac{T_{i c}}{T_{i}}\right)^{8.5}\left(1-\frac{1-X_{i}^{2}}{1-X_{e}^{2}} \frac{\mu_{i}}{\mu_{e}}\right)\right] \leqslant \frac{2}{5}
$$

At the interface, $T_{i}=T_{i c}$, and condition (26) for stability reduces to

$$
\frac{1}{4.25}\left(\frac{1-X_{i}^{2}}{1-X_{e}^{2}} \frac{\mu_{i}}{\mu_{e}}\right) \leqslant \frac{2}{5}
$$

and when this is taken with the equality sign, it is equivalent to condition (11) of Hoyle and Lyttleton. Now if the values of $\mu_{i}$ and $\mu_{e}$ are such that

$$
\left(\frac{1-X_{e}^{2}}{1-X_{i}^{2}}\right) \frac{\mu_{e}}{\mu_{i}}<\frac{5}{8.5}=0.588,
$$

the radiative equilibrium will be unstable on the internal side of the interface. However, as we leave the interface, $\left(T_{i c} / T_{i}\right)^{8.5}$ decreases so rapidly that, after we have gone only a little distance, condition (26) will again be satisfied. We can, accordingly, expect that the extent of the unstable region resulting from the violation of equation (27) will be very small indeed.

On the other hand, as we have already seen, a discontinuity of $\mu$ (with $\mu_{i}>\mu_{e}$ ) is going to provide a very effective barrier to convection. Thus we must expect that the energy available for convection on the internal side of the interface will soon be used up by the mixing of two narrow regions on both sides of the interface.

As an example, let us take $\mu_{e}=1, X_{e}=\frac{1}{3}$, and $\mu_{i}=2, X_{i}=0$, and suppose that the discontinuity of $\mu$ occurs at $r_{c}=0.9 R$. In that case, using condition (26) and equations (21) and (5), we find that the unstable region extends to the point where $P, T$, and $\rho$ have the values

$$
P_{i}^{*}=1.1616 P_{i c} ; \quad T_{i}^{*}=1.0706 T_{i c} ; \quad \text { and } \quad \rho_{i}^{*}=1.0850 \rho_{i c} .
$$

Introducing into equation (25) the numerical value of $\beta$ corresponding to this case and integrating, we get

$$
r_{c}-r^{*}=0.01812 \frac{r_{c}}{R}\left(R-r_{c}\right)
$$

so that the extent of the unstable region is of the order of 1-2 per cent of the external region. In the particular case considered $\left(r_{c}=0.9 R\right)$,

$$
r^{*}=0.89834 R \text {. }
$$

To compute the mass $\Delta m_{i}$ of the unstable region, we can assume that $\rho$ is constant in it and equal to $\rho_{i c}$, so that, using equations (18), (20), and (10), we can write the ratio of $\Delta m_{i}$ to the mass $M_{e}$ of the external part as

$$
\frac{\Delta m_{i}}{M_{e}}=\frac{\mu_{i}}{\mu_{e}}\left(\frac{1}{\xi_{c}}-1\right)^{3.25} \frac{\left(\xi_{c}^{3}-\xi^{* 3}\right)}{3 f\left(0, \xi_{c}\right)},
$$


where $\xi=r / R$ and $f\left(0, \xi_{c}\right)$ is the function tabulated by S. Chandrasekhar. ${ }^{7}$ In our example, with the value 0.000014 of $f(0,0.9)$ given by S. Chandrasekhar, formula (31) gives $\Delta m_{i} / M_{e}=0.153$.

The solutions $T / T_{e c}, P / P_{e c}$, and $\rho / \rho_{e c}$ in the vicinity of the interface are represented in Figure 1 (full curves).

4. Energy available in the unstable region and resulting motion and mixing.-Consider, next, an element of matter of unit mass situated at the base of the unstable region. If a

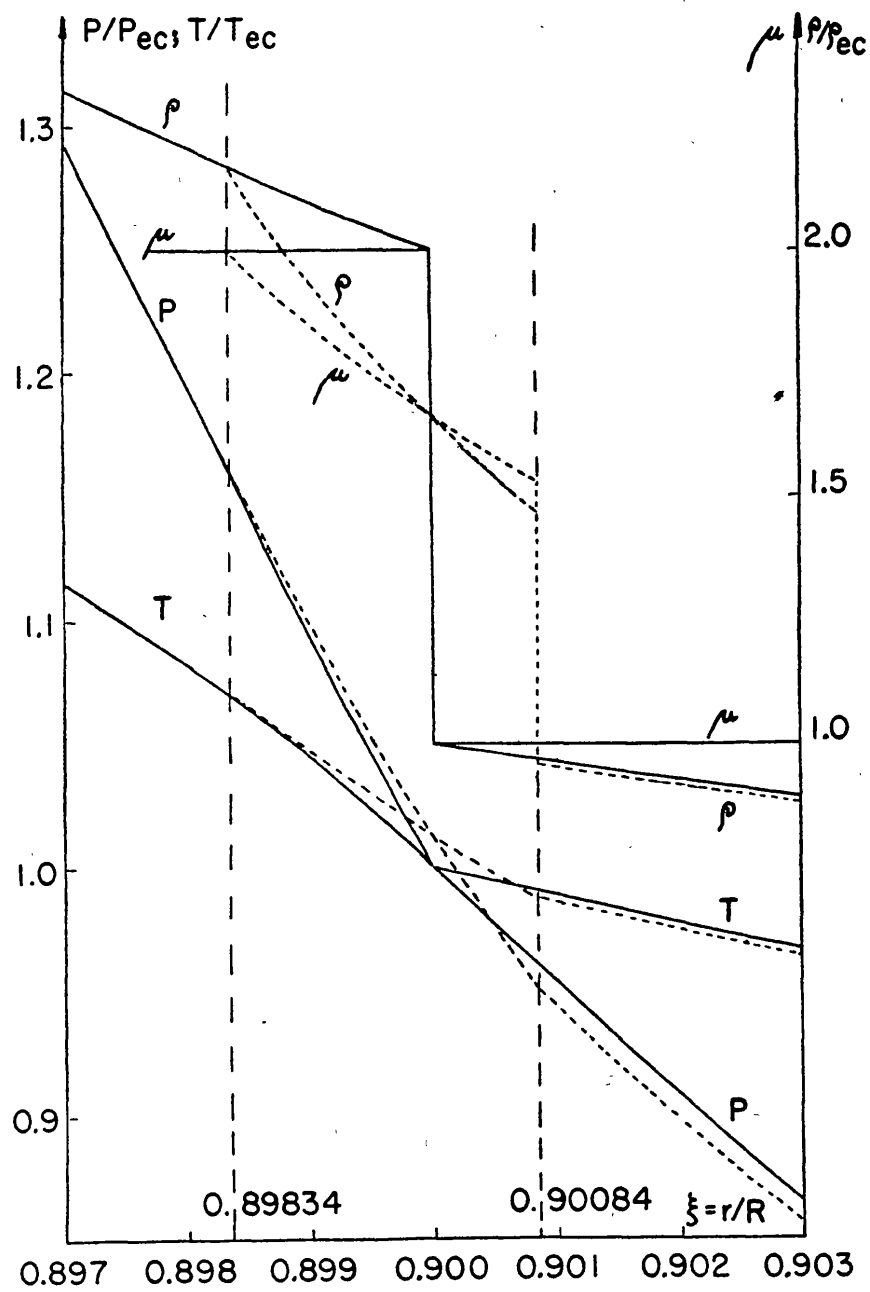

FIG. 1.-Distribution of the physical conditions at a discontinuity of $\mu$ in the stable (dotted curves) and unstable states (full curves).

small adiabatic displacement outward is given to it, its speed outward will increase all through the unstable region. Neglecting friction, we can express its kinetic energy, on reaching the interface, in the form

$$
\frac{1}{2} v^{2}=\frac{P_{i c}}{\rho_{i c}} \int_{\left(P_{i}^{*} / P_{i c}\right)}^{1}\left[\left(\frac{\rho_{i c}}{\rho_{i}}\right)-\left(\frac{\rho_{i c}}{\rho_{a d}}\right)\right] d\left(\frac{P_{i}}{P_{i c}}\right)
$$

where

$$
\rho_{a d}=\rho_{i}^{*}\left(\frac{P_{i}}{P_{i}^{*}}\right)^{3 / 5}=\rho_{i c}\left(\frac{\rho_{i}^{*}}{\rho_{i c}}\right)\left(\frac{P_{i c}}{P_{i}^{*}}\right)^{3 / 5}\left(\frac{P_{i}}{P_{i c}}\right)^{3 / 5}
$$

${ }^{7}$ Op. cit., p. 302 . 
For the example studied in $\S 3$, the values of the different constant ratios in equation (33) have been given, and the values of $\rho_{i} / \rho_{i c}$ and $P_{i} / P_{i c}$ can be obtained from the solutions given there or can be taken directly from Figure 1. Evaluating the integral, we find that

$$
\frac{1}{2} v^{2}=0.000405 \frac{P_{i c}}{\rho_{i c}}=2.7 \times 10^{-4}\left(n \frac{3}{2} k T_{i c}\right),
$$

where $n$ is the number of the particles contained in the element considered. Equation (34) shows that the available energy in the unstable region is very small, compared to the internal energy of that region, which is itself a small quantity. It is, therefore, difficult to see how any large-sćale motion can result from the instability in the small region bordering on $r_{c}$.

An elementary computation also shows that the time required by a small element to go across the unstable region is of the order of

$$
\Delta t \simeq \frac{10^{2}}{\sqrt{\bar{\rho}}} \text { seconds }
$$

where $\bar{\rho}$ is the mean density, in grams, of the star considered.

Now a rising element of gas will arrive at the interface with a finite kinetic energy of the order of magnitude given by expression (34). Using the corresponding equation (32) and the relation (20) between $\rho_{e}$ and $P_{e}$, it is easy to determine the pressure $P_{e}^{0}$ corresponding to the level $\xi^{0}$ in the external part where that kinetic energy will be used up. We find $P_{e}^{0}=0.9996 P_{e c}$ and $\xi^{0}=0.90001$. If we denote by $\Delta m_{e}$ the mass of the layer comprised between $\xi_{e}$ and $\xi^{0}$, its ratio to the mass $\Delta m_{i}$ of the unstable region is of the order of

$$
\frac{\Delta m_{e}}{\Delta m_{i}} \simeq \frac{\rho_{e c}}{\rho_{i c}} \frac{\xi^{0}-\xi_{c}}{\xi_{c}-\xi^{*}}=0.0027 .
$$

If we now consider a downward motion, a small element initially at the internal side of the interface will stop somewhere between the points $\xi^{*}$ and $\dot{\xi}_{s}$, where the following relations hold, respectively,

and

$$
\frac{1}{T_{i}^{*}} \frac{d T_{i}^{*}}{d r}=\frac{2}{5} \frac{1}{P_{i}^{*}} \frac{d P_{i}^{*}}{d r}
$$

$$
\frac{P_{i s}}{P_{i c}}=\left(\frac{T_{i s}}{T_{i c}}\right)^{5 / 2} .
$$

From our discussion in the preceding paragraph it follows that, when $\mu$ is assumed to change discontinuously at a point in violation of condition (27), turbulence in a small region surrounding that point will immediately result. This resulting turbulence will lead to a small amount of mixing of the internal and the external parts. And if, as we shall show, a neighboring stable state can be reached in consequence of this turbulence, it is not really relevant to our problem to describe in detail the exact changes which will lead to that stable state. However, in a general way, it is apparent that the first step will be a mixing of two regions whose masses are of the order of $\Delta m_{e}$ and $\Delta m_{i}$ with the formation of an intermediate region of mass $a \Delta m_{e}+b \Delta m_{i}$ and mean molecular weight

$$
\mu_{m} \simeq \mu_{i}\left\{1-\left(\frac{\mu_{i}}{\mu_{e}}-1\right) \frac{a \Delta m_{e}}{b \Delta m_{i}}\right\}
$$

where $a$ and $b$ are two constants of order unity. In the example considered, $\mu_{m}=1.995$ if $a=b=1$. The radiative solutions for these regions, characterized, respectively, by $\mu_{i}, \mu_{m}$, and $\mu_{e}$, can be obtained and the radiative instability at each interface examined. 
It will again be seen that, in the intermediate part, there will be a small region around the points defined by the corresponding relations (38) and (37) which will be little affected by mixing at this stage, and the extent of the region affected in the internal part will again be determined by the same analogous relations. The changes in $\mu$ which will result from the corresponding mixing can be estimated as before, and the process can be continued a step further. In this manner a stable state can be reached, and the time required to attain it can be estimated as follows:

At a given stage the time required for a single "turnover" of the unstable regions may be taken to be of the same order as that given by equation (35). A hundred such turnovers would probably be more than sufficient to mix the relevant regions thoroughly. If a thousand such steps are necessary to bring the regions to a stable state, a total time of the order of $1 / \sqrt{\bar{\rho}}$ years will be required. For all reasonable values of $\bar{\rho}$ this is such a short time, as compared to the time required for the building-up of an appreciable discontinuity of $\mu$ (either by exhaustion of hydrogen, by nuclear reactions, or by accretion of interstellar hydrogen) that we can safely consider that the readjustment takes place practically instantaneously!

5. Final state of equilibrium.-What we have said in $\$ 4$ of the detailed manner in which a stable state will be reached is sufficient to show that at each step there will be a number of small regions (equal to the number of discontinuities of $\mu$ ), each little affected by the subsequent mixing and comprised between points $\xi^{*}$ and $\xi_{s}$, where the relations corresponding to (37) and (38) are satisfied. As the number of discontinuities in $\mu$ increases, these two points, $\xi^{*}$ and $\xi_{s}$, in each region of constant $\mu$ will tend to come closer and closer. At the limit, where the variation of $\mu$ can be considered as continuous, relations (37) and (38) will hold at each point, so that, if we denote by a suffix $t$ the values of the variables in that region, we have

and

$$
\frac{1}{T_{t}} \frac{d T_{t}}{d r}=\frac{2}{5} \frac{1}{P_{t}} \frac{d P_{t}}{d r}
$$

$$
\frac{P_{t}}{P_{t c}}=\left(\frac{T_{t}}{T_{t c}}\right)^{5 / 2}
$$

where the suffix $c$ here refers to the values of the variables at the exterior limit of the region of variable $\mu$.

If we assume that this intermediate region of variable $\mu$ is also in radiative equilibrium, equation (14) should hold in it; and, comparing it with equation (39), we obtain

$$
C\left(1-X_{t}^{2}\right) \mu_{t}=\frac{P_{t}^{2}}{T_{t}^{8.5}}=\frac{2}{5},
$$

or, using equation (40),

$$
\left(1-X_{t}^{2}\right) \mu_{t}=\frac{2}{5 C} \frac{T_{t c}^{8.5}}{P_{t c}^{2}}\left(\frac{P_{t}}{P_{t c}}\right)^{7 / 5}
$$

With equation (41), condition (17) for the stability of the radiative equilibrium in that region reduces to

$$
\frac{d \log \mu_{t}}{d \log P} \geqslant 0
$$

But, according to equation (42), $\mu_{t}$, in fact, increases with $P$, so that condition (43) is satisfied and our assumption about the stability of radiative equilibrium in that region is confirmed. This transition region of variable $\mu$ will be limited by the fact that when $\mu_{t}$ 
has been reduced to a certain value, $\mu_{t c}$, such that equation (27) with $\mu_{i}=\mu_{t c}$ is verified with the sign of equality, the discontinuity of $\mu$ is no longer able to render the radiative equilibrium unstable. If $\mu_{e}=1, X_{e}=\frac{1}{3}$, we find that $\mu_{t c}$ and $X_{t c}$ must have the values $X_{t c}=0.1031$ and $\mu_{t c}=1.528$. These are, of course, equivalent to the limiting values of Hoyle and Lyttleton.

When $\mu_{t}$ varies from its value 1.528 to its value $2,\left(1-X_{t}^{2}\right)$ varies from 0.99 to 1 ; and in a first approximation we can neglect this factor, so that equation (42) immediately gives the variation of $\mu$ in the form

$$
\frac{\mu_{t}}{\mu_{t c}}=\left(\frac{P_{t}}{P_{t c}}\right)^{7 / 5}
$$

From equations (5) and (40), we obtain

$$
\frac{\rho_{t}}{\rho_{t c}}=\left(\frac{P_{t}}{P_{t c}}\right)^{2}
$$

The integration of equation (13) then gives

$$
1-\frac{P_{t c}}{P_{t}}=\frac{G M \mu_{t c}}{\Re T_{t^{*}}}\left(\frac{1}{r}-\frac{1}{r_{c}}\right) .
$$

The solution allowing for the variation of the factor $\left(1-X_{t}^{2}\right)$ in this transition region can, of course, be obtained by eliminating $X_{t}$ between equations (4) and (42) and then proceeding as above. But we shall not consider such refinements here.

In the external part $\left(\mu=\mu_{e}\right)$, the solution is the same as before, and it is, of course, stable.

In the internal part $\left(\mu=\mu_{i}\right)$, integrating equation (14) and taking into account the fact that all the variables must be continuous at the interface, $r^{*}$, where the variation of $\mu_{t}$ ceases, we obtain

$$
C\left(1-X_{i}^{2}\right) \mu_{i} \frac{P_{i}^{2}}{2}=\frac{T_{i}^{8.5}}{8.5}\left[1+0.7\left(\frac{T_{i}^{*}}{T_{i}}\right)^{8.5}\right] .
$$

The gradient of temperature in this region can be expressed in the form

$$
\frac{d T_{i}}{d r}=-\frac{\mu_{i} G M}{4.25 \Re}\left[1+0.7\left(\frac{T_{i}^{*}}{T_{i}}\right)^{8.5}\right] \frac{1}{r^{2}}
$$

Using the value of $P_{i}^{2} / T_{i}^{8.5}$ given by equation (47), the criterion of stability (16) can be written

$$
\frac{2}{5}-\frac{1.4}{8.5}\left[1-\left(\frac{T_{i}^{*}}{T_{i}}\right)^{8.5}\right] \leqslant \frac{2}{5}
$$

and we see that this condition is just satisfied with the sign of equality at the interface. As we go further inside, $\left(T_{i}^{*} / T_{i}\right)^{8.5}$ decreases, and the requirement (49) will be met with the sign of inequality. Thus we can picture the star in its final state as being composed of the three parts we have described, all of which are in stable radiative equilibrium.

It may be noted that when $\mu_{e}$ and $\mu_{i}$ are given, the extent of the intermediate region, where $\mu$ varies continuously, is determinate, for, $\mu_{e}$ being known, equation (27) taken with the sign of equality can be solved for $\mu_{t c}$ and, by equations (44) and (46), we have

$$
\frac{r_{c}}{R}-\frac{r^{*}}{R}=\left[1-\left(\frac{\mu_{t c}}{\mu_{i}}\right)^{5 / 7}\right] \frac{\mu_{e}}{\mu_{t c}} \frac{1}{4.25} \frac{r^{*}}{R}\left(1-\frac{r_{c}}{R}\right)
$$

since $T_{t c}$ must be equal to $T_{e c}$ and its value can be obtained from equation (18). 
Thus, if we choose the values of $\mu_{i}, \mu_{e}$, and $r_{c}$, the solution is completely determined in the envelope, and the problem of fitting it to a central part without singularity can be solved in the usual way. We shall not concern ourselves here with the actual mass"luminosity radius relation appropriate for these models, except to point out that the relevant relation will be practically the same whether we consider the change in $\mu$ as taking place discontinuously and ignore the effect of the small convective unstable region just interior to the interface, or whether we treat it more rigorously with the introduction of a transition region of variable $\mu$. We shall illustrate this numerically for the 'example we have considered.

Suppose that the general problem has been solved for the case in which $\mu$ has been assumed to change discontinuously from its value $\mu_{e}$ to $\mu_{i}$ at $r_{c}=0.9 R$ and that the configuration has a mass $M$, luminosity $L$, and radius $R$. The effect of the instability in the small region interior to $r_{c}$ will, of course, be that the star will rapidly evolve toward the state with a transition region of variable $\mu$ as we have already described. During such an adjustment, the total mass will, of course, remain constant; and, to the extent that the energy sources are in the deep interior, the luminosity will also remain constant. What will happen then is that the radius will change slightly. To estimate the extent of this change in $R$ which will result from the redistribution of $\mu$, we shall consider a second configuration with the same luminosity, in which the inner limit $r^{*}$ of the transition region is at the same place at which the convectively unstable region in the first configuration ends. We shall further assume that the temperatures in the two configurations are also the same at the two points mentioned. Under these conditions, the solutions interior to $r^{*}$ in the two configurations will be identical. And if the problem for the interior on the approximate assumption of a discontinuous $\mu$ has been solved for the first configuration, the problem can be considered as solved for the second configuration as well. On the other hand, for $r>r^{*}$ we can express the temperature $T$ in the two configurations considered in terms of the radius $r$ and the parameter $T^{*}$. And, since $r^{*}$ and $T^{*}$ have been assumed to be the same, we can eliminate the temperature from the relations appropriate for the two models applied at the end of the external regions $(\mu=1)$. In this way we obtain a relation between the radii $R$ and $R^{\prime}$ of the two configurations and the value $r_{c}=0.9 R$ and $r_{c}^{\prime}$, which defines the outer extent of the transition region in the second configuration. Equation (50) provides another relation, so that we can solve for both $r_{c}^{\prime}$ and $R^{\prime}$. We get

$$
r_{c}^{\prime}=0.90084 R \quad \text { and } \quad R^{\prime}=0.99973 R \text {. }
$$

The last relation shows that, for all practical purposes, $R$ can be considered as unchanged.

If we should now integrate equation (7) from $r^{*}$ outward in both configurations, we should find that the masses of the external parts are not quite the same. But, remembering the order of magnitude of the external mass and comparing the new distribution of density (dashed curve in Fig. 1) with the old one, it is evident that the difference, as compared to the total mass, cannot be greater than one part in $10^{6}$. In other words, the masses will be the same to a precision even higher than that in which the numerical integrations are generally carried out for the interior!

Thus, contrary to what Hoyle and Lyttleton have said, with a discontinuity of $\mu$ in the external layers not satisfying condition (27), we can always construct a stable state of equilibrium very near the one obtained by ignoring this condition altogether. And, as we have further seen, the final stable state will be reached in a relatively short time by turbulent mixing of a very narrow zone, which has the effect essentially only of "smoothing" the discontinuity. No large-scale motions of the kind imagined by Hoyle and Lyttleton can intervene.

So far we have treated only one example, where $\mu_{i}=2$ and $\mu_{e}=1$, but it is apparent that the conclusions will remain valid for all reasonable discontinuity of $\mu$. When the pressure of radiation is not negligible, the algebra becomes somewhat more complicated, 
but, unless it becomes a very large part of the total pressure, it does not introduce any further novel features.

6. Discontinuity of $\mu$ in the interior of the star.-We shall limit ourselves again to the case in which the pressure of radiation is negligible. But, in contrast to our earlier discussion, we must allow for the variation of $m(r)$, and an analytical treatment becomes impossible. However, the use of an effective polytropic index, as defined by equation (12), and of the homology invariant variables, ${ }^{8}$

and

$$
U=\frac{4 \pi \rho r^{3}}{m(\boldsymbol{r})}=\frac{d \log m(\boldsymbol{r})}{d \log (\boldsymbol{r})}
$$

$$
V=\frac{2}{5} \frac{G m(r) \rho}{r P}=-\frac{2}{5} \frac{d \log P}{d \log r}
$$

simplifies the discussion considerably. The use of these variables is suggested since, in the fitting-together of two solutions, the continuity of $U$ and $V$ automatically implies the continuity of $m(r), P$, and $T$ across the interface. If different values of $\mu$ are assumed in the different regions, then it is the continuity of $U / \mu$ and $V / \mu$ across the interface which should be required. Since the generation of energy is strongly concentrated toward the center, the distribution of physical conditions in an extensive region of the star from the surface inward is given by a point-source solution. For a given total mass $M$, we have a whole family of such solutions, which can be arranged and discussed in the $(U, V)$-plane very much in the same way as the polytropic solutions corresponding to a given index $n .^{9}$

The differential equations for the variations of $U, V$, and $n$ along a point-source solution can be obtained easily from definitions (12), (51), and (52) and from equations (3) and (7). They are

and

$$
\begin{aligned}
& \frac{d \log U}{d \log r}=3-U-\frac{5}{2} \frac{n}{n+1} V-\frac{5}{2} V \frac{d \log \mu}{d \log P} \\
& \frac{d \log V}{d \log r}=U+\frac{5}{2} \frac{1}{n+1} V-1-\frac{5}{2} V \frac{d \log \mu}{d \log P}
\end{aligned}
$$

If $\mu$ is constant, these equations reduce to

and

$$
\frac{d \log (n+1)}{d \log r}=U-\frac{5}{2}\left(\frac{6.5-2 n}{n+1}\right) V+\frac{5}{2} V \frac{d \log \mu\left(1-X^{2}\right)}{d \log P} \text {. }
$$

$$
\frac{d \log V}{d \log r}=U+\frac{5}{2} \frac{1}{n+1} V-1
$$

$$
\frac{d \log (n+1)}{d \log r}=U-\frac{5}{2}\left(\frac{6.5-2 n}{n+1}\right) V .
$$

Suppose that we go toward the center of a star along one of the point-source solutions labeled by a $Q$ and that we arrive at a point $r_{c}$, where $\mu$ jumps from its value $\mu_{e}$ to a value

${ }^{8}$ Cf. Chandrasekhar, op. cit., p. 352.

${ }^{9} \mathrm{I}$ am indebted to Dr. S. Chandrasekhar for allowing me the use of his tabulation of the functions $U$ and $V$ for a whole family of point-source solutions. In Dr. Chandrasekhar's tabulations the pointsource solutions have all been conveniently reduced in a standard system of nondimensional variables (cf. Mrs. Harrison, $A$. J. J., 103, 193, 1946, particularly eqs. [23]-[27]). 
$\mu_{i}$. At this point the effective polytropic index will have a certain value $n_{e}$ and, by condition (11) of Hoyle and Lyttleton, we can determine the value of $n_{i}$. Let us assume, first, that the discontinuity of $\mu$ and the value of $n_{e}$ are such that $n_{i}=\frac{3}{2}$. Now suppose that the conditions of fit to a polytropic solution $E_{3 / 2}$ are satisfied at $r_{c}$ (i.e., that in the $[U, V]$-plane, the point of co-ordinates $\mu_{i} U_{c} / \mu_{e} \mu_{i} V_{c} / \mu_{e}$ lies on $\left.E_{3 / 2}\right)$. There is no loss of generality in making this assumption, since it is always possible to find such a point $r_{c}$ on any of the point-source solutions. However, the fact that $n_{i}$ is equal to $\frac{8}{2}$ just on the internal side of the interface does not mean that this is the starting-point of a convective region.

To examine the stability of the radiative gradient in the region interior to $r_{c}$, we let $n=n_{i}=\frac{8}{2}$ in equation (58) and obtain for the variation of $n$ just past the interface the equation

$$
\frac{d \log (\dot{n}+1)}{d \log r}=U-\frac{r}{2} V .
$$

Thus, if the representation of the point $r_{c}$ in the $(U, V)$-plane falls above the line

$$
2 U-7 V=0 \text {, }
$$

$n$ increases again above its value $\frac{3}{2}$ as we leave the interface toward the interior, and the radiative gradient continues to be stable. It may be noted, however, that the radiative solution which we should use to extend our solution past the discontinuity of $\mu$ need not belong to the usual point-source solutions, since in this case $P, \rho$, and $T$ need not tend simultaneously to zero at some value of $r$.

It is now seen that line (60) lies very close to the locus of the points on the radiative solutions where $n$ becomes equal to $\frac{3}{2}$. Since it is only between the line (60) and the locus of the points $n=\frac{3}{2}\left(\mu_{i}>\mu_{e}\right)$ that condition (11) of Hoyle and Lyttleton can lead to a fitting with a convective core, it is evident that the models obtained in this way cannot differ very much from the one fitted at the points $n=\frac{3}{2}$. However, condition (11) has the further consequence that, since on any. of the point-source solutions $n_{e}$ will remain very close to $\frac{8}{2}$ in the region where the fit to a convective core is possible, the only permissible discontinuities of $\mu$ at the interface with a convective core are going to be very small (cf. eq. [11]). In fact, all the resulting models would be very close to Cowling's solution $\left(\mu_{i}=\mu_{e}\right)$.

We have considered the case in which the discontinuity of $\mu$ is such that $n_{i}$ according to condition (11) is just equal to $\frac{8}{2}$. But, since $n_{e}$ has its maximum value (3.25) in the external layers, $n_{i}$ will always be smaller than $\frac{3}{2}$, say, $\left(\frac{8}{2}-\epsilon\right)$, no matter where the discontinuity takes place, provided that $\mu_{i} / \mu_{e}>1.53$. The variation of $n$ on the internal side of the interface will then be given by

$$
\frac{d \log (n+1)}{d \log r}=U-\frac{17.5+10 \epsilon}{5-2 \epsilon} V .
$$

As $\epsilon$ increases, the region of the $(U, V)$-plane, where $d n / d r$ is negative, increases also; and, although $n_{i}$ becomes smaller than $\frac{3}{2}$ just at the interface, it will start increasing after that, and in many cases radiative equilibrium will soon re-establish itself. The situation is therefore very similar to what we have described under analogous conditions in the stellar envelope $\left(r / R>\frac{1}{2}\right)$. We may accordingly expect that, in our present context also, there will be a rapid readjustment to a transition region, in which $\mu$ will vary. To determine the law of variation of $\mu$ in the resulting transition region we argue as follows:

For any given point, $\boldsymbol{r}_{c}$, the maximum discontinuity in $\mu$ which is compatible with the stability of the radiative gradient $\left(n_{i}=\frac{3}{2}\right)$ just past the interface will be given by condition (11) with the equality sign. If $\mu$ remains constant after crossing the interface and if we should be above line $(60), n_{i}$ will start increasing again for decreasing $r$. Indeed, ac- 
cording to equations (58), (51), and (52), the increase in $n_{i}$ which will take place when $r$ decreases by $\Delta r$ will be given by

$$
\frac{2}{5} \Delta n_{i}=(U-3.5 V) \frac{\Delta r}{r_{c}}=\Delta \log m(r)+\frac{7}{5} \Delta \log P .
$$

At $r=r_{c}-\Delta r$, therefore, $n_{i}=\frac{3}{2}+\Delta n_{i}>\frac{3}{2}$, and we can again have a small discontinuity of $\mu$ of amount $\Delta \mu$, which will reduce $n_{e}=\frac{3}{2}+\Delta n_{i}$ to $\frac{3}{2}$ again, according to condition (11). Using condition (11), we obtain the following relation between $\Delta n_{i}$ and $\Delta \mu$

$$
\frac{\Delta \mu}{\mu}=\frac{2}{5} \Delta n
$$

if we neglect the factor $\left(1-X^{2}\right)$; as we have already seen in our earlier discussion in $\$ \$ 4$ and 5 , the factor $\left(1-X^{2}\right)$ has only a very small influence on the final results. Using relation (62), we find that equation (63) becomes

$$
\frac{\Delta \mu}{\mu}=\Delta^{k} \log m(r)+\frac{7}{5} \Delta \log P \text {. }
$$

We can repeat this process until we have reached the pre assigned value $\mu_{i}$ for the core. Making the various steps $\Delta r$ smaller and smaller, we shall obtain, in the limit $\Delta r=0$, a region throughout which

$$
n_{i}=\frac{3}{2}
$$

Further in this region, $\mu$ will vary continuously accordingly to the law

$$
\mu=C_{1} m(r) P^{7 / 5} \text {. }
$$

According to equation (65), we also have

$$
T=C_{2} P^{2 / 5} .
$$

Apart from the factor $m(r)$ (the variation of which cannot be neglected in our present context), relations (66) and (67) are the same as those obtained in $\$ 5$ (eqs. [40] and [44]) by considering in detail the process of mixing.

The differential equations (53) and (54) for $U$ and $V$ in the transition region of variable $\mu$ are therefore (cf. eq. [66])

and

$$
\frac{d \log U}{d \log r}=3-5 V
$$

$$
\frac{d \log V}{d \log r}=2 U-\frac{5}{2} V-1
$$

Combining these two equations, we have

$$
\frac{d V}{d U}=\frac{V}{U} \frac{2 U-\frac{5}{2} V-1}{3-5 V}
$$

The general nature of the solutions of equation (70) can be readily pictured by drawing the system of isoclinical curves in the $(U, V)$-plane, along which $d V / d U$ is constant. These isoclinical curves are a series of hyperbolas and are shown in Figure 2. The short lines (of constant slope) drawn across each curve represent the directions in which the solutions of equation (70) will cross this curve. From the arrangement of the isoclinical curves in Figure 2, it is seen that the solution-curves of equation (70) spiral around the 
point $U=1.25$ and $V=0.6$; at this point both the numerator and the denominator of the quantity on the right-hand side of equation (70) vanish. This singular point of equation (70) corresponds to the following singular solution of equations (1)-(7) when relations (66). and (67) are satisfied:

$$
\begin{gathered}
P=C_{1} r^{-3 / 2}, \quad \rho=C_{2} r^{-7 / 4}, \quad T=C_{3} r^{-3 / 5}, \\
\mu=C_{4} r^{-17 / 20}, \quad \text { and } \quad m(r)=C_{5} r^{5 / 4}
\end{gathered}
$$

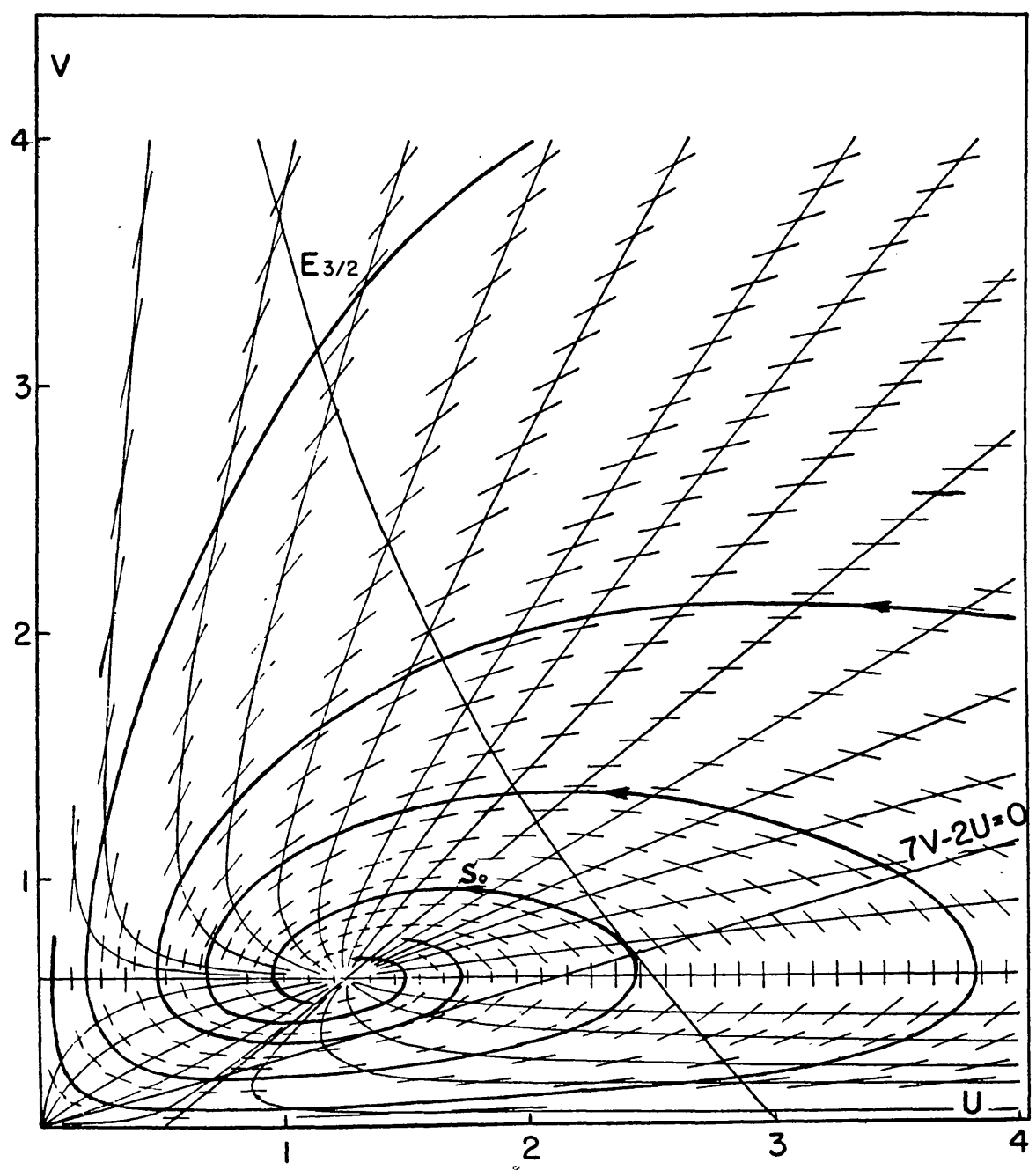

Frg. 2.-General run of the spiral solutions in a region where $\mu$ varies continuously according to the law $\mu \propto m(r) P^{7 / 5}$.

here $C_{1}, C_{2}$, etc., are constants. All the other solutions asymptotically tend to this singular solution as we approach the surface $(r \rightarrow \infty, m[r] \rightarrow \infty)$, and along them the radius increases in the direction indicated by the arrows.

Along any one of the spiral solutions we have (cf. eq. [66])

$$
\frac{d \log \mu}{d \log P}=U-\frac{7}{2} V \text {. }
$$

Thus $\mu$ increases with decreasing $r$ above line (60); while below this line $\mu$ decreases with decreasing $r$. According to the criterion of stability (9) and equation (67), we conclude 
that the radiative gradient will be unstable below the line (60), and therefore these portions of the spiral solutions should not be used in physical models.

We shall now show how, with the help of the solutions sketched in Figure 2, we can construct stellar models in which $\mu$ is assigned certain values for the $e_{A}$ envelope $\left(\mu=\mu_{e}\right)$ and the core $\left(\mu=\mu_{i}\right)$ with possible transition zones in which $\mu$ is variable according to law (66). Suppose, now, that the region of $\mu=\mu_{e}$ (point-source solution) extends down to $r=r_{c}$, where $U=U_{c}$ and $V=V_{c}$. At this point $\left(U_{c}, V_{c}\right), \mu$ can jump to a value $\mu_{t}$ given by condition (11) with $n_{i}=\frac{8}{2}$. If that point is above the line (60), $\mu$ can then continue to increase according to equation (66). We must now follow, in the sense of decreasing $r$, the spiral solution starting at the point $\left(\left[\mu_{t} / \mu_{e}\right] U_{c},\left[\mu_{t} / \mu_{e}\right] V_{c}\right)$, until $\mu$ reaches its value $\mu_{i}$, say, at the point $\left(U^{*}, V^{*}\right)$. As we continue the solution further, $\mu$ must remain constant. If the point $\left(U^{*}, V^{*}\right)$ is still above line $(60)$, then we, must continue with a radiative solution which ultimately will become unstable deeper in the star, owing to the concentration of energy sources. When this occurs, the solution should be continued by an $E_{3 / 2}$ solution, and the conditions of fit there will permit us to fix the point-source solution to be used in the external part of the star.

TABLE 1

\begin{tabular}{|c|c|c|c|c|c|c|c|}
\hline \multirow{2}{*}{$\mu_{i} / \mu_{c}\left(\mu_{e}=1\right)$} & \multirow{2}{*}{$\mu t / \mu_{e}$} & \multirow{2}{*}{$m_{e} / M$} & \multirow{2}{*}{$r_{e} / R$} & \multirow{2}{*}{$m_{t} / M$} & \multirow{2}{*}{$r_{t} / R$} & \multicolumn{2}{|c|}{ HARRISON* } \\
\hline & & & & & & $m_{c} / M$ & $r_{c} / R$ \\
\hline $\begin{array}{r}\text { Cowling } 1 . \\
1.055 \ldots \\
1.147 \ldots \\
1.248 \ldots \\
1.483 \ldots \\
1.8 \ldots \ldots \\
2.0 \ldots \ldots\end{array}$ & $\begin{array}{l}1.144 \\
1.206 \\
1.276 \\
1.315 \\
1.320\end{array}$ & $\begin{array}{r}0.150 \\
.168 \\
.189 \\
.181 \\
.154 \\
.123 \\
0.104\end{array}$ & $\begin{array}{r}0.171 \\
.171 \\
.164 \\
.150 \\
.117 \\
.086 \\
0.072\end{array}$ & $\begin{array}{r}0.206 \\
.230 \\
.240 \\
.230 \\
0.214\end{array}$ & $\begin{array}{r}0.170 \\
.165 \\
.143 \\
.116 \\
0.103\end{array}$ & $\begin{array}{r}0.150 \\
.143 \\
.131 \\
.119 \\
.095 \\
.083 \\
0.081\end{array}$ & $\begin{array}{r}0.171 \\
.161 \\
.146 \\
.132 \\
.108 \\
.091 \\
0.087\end{array}$ \\
\hline
\end{tabular}

*Ap. J., 100, 343, 1944.

The case of immediate physical interest is, however, one in which the convective core starts just at the end of the transition region of variable $\mu$, since it is in the convective core that the principal change in the chemical composition occurs. Further in the convective core $\mu$ must be uniform on account of mixing. In this case, since $n$ must decrease at the end of the zone of transition, the point $\left(U^{*}, V^{*}\right)$, where $\mu$ reaches its value $\mu_{i}$, must lie under or on line (60). On the other hand, we have seen that we can use the spiral solutions only above this line. Thus the only spiral solution which will lead to a convective core starting at the end of the zone of transition is the one which intersects $E_{3 / 2}$ at the same point as the straight line (60). It can be shown that at this point the spiral solution and $E_{3 / 2}$ have the same tangent. This particular spiral solution is shown in Figure 2. Using this solution $S_{0}$ tangential to $E_{3 / 2}$, we constructed a few models composed of a point-source envelope followed by a region of transition which ends in a convective core. For a given change of $\mu\left(\mu_{e}, \mu_{i}\right)$ we must find on one of the point-source solutions a point $U_{c}, V_{c}, n_{c}$, such that its co-ordinates, multiplied by $\mu_{t} / \mu_{e}$ (where $\mu_{t}$ is given by condition [11] of Hoyle and Lyttleton with $\left.n_{e}=n_{c}, n_{i}=\frac{3}{2}\right)$, represent a point on the spiral solution $S_{0}$. Furthermore, as we go along that solution (decreasing $r$ ), $\mu$ increases from its value $\mu_{t}$, and it should just reach the value $\mu_{i}$ at the point of contact with $E_{3 / 2}$. In general, for given $\mu_{e}$ and $\mu_{i}$, we shall have to interpolate between the various pointsource solutions. It is, therefore, more convenient to start from a particular point-source solution and determine the corresponding variation of $\mu$ which permits us to fit it to a 
convective core with a transition zone. It is in this way that the results given in Table 1 were obtained. The mass and radius of the convective core are denoted by $m_{c}$ and $r_{c}$ and those of the convective core, plus the zone of transition, by $m_{t}$ and $r_{t}$. It will be noted that the second model has no zone of transition, and it corresponds to the case where the condition of Hoyle and Lyttleton leads directly to a solution. In fact, the corresponding discontinuity of $\mu$ is already close to the maximum compatible with this condition alone.

In the last two columns we have given the masses and radii of the convective cores derived by Schönberg and Chandrasekhar (op. cit) and Mrs. Harrison ${ }^{10}$ in their discussion of the "generalized Cowling model." As we have already stated, in the discussions carried out by these writers $\mu$, was assumed to jump discontinuously from $\mu_{e}$ to $\mu_{i}$ at the surface of the convective core, with a corresponding discontinuity of density. It will be seen that the general sense of the evolution derived by these writers is in general agreement with our more rigorous treatment of the same problem.

Finally, we may note that for the model $\mu_{i} / \mu_{e}=2$, we find $L=2.78 L$ (Cowling's model) and $R=2.57 R$ (Cowling's model). They are somewhat greater than those obtained by M. Schönberg and S. Chandrasekhar, who found $L=1.41 L$ (Cowling's model) and $R=1.65 R$ (Cowling's model). The central condensation is also greater, and the ratio $\rho_{v} / \bar{\rho}$ becomes equal to 390 instead of 179 . But the differences between the two models become less for smaller ratios of $\mu_{i} / \mu_{e}$.

It is seen from Table 1 that if we wish to regard these models as a sequence of evolution, the mass of the convective core starts by increasing. But, as soon as the chemical composition has been altered appreciably, its mass decreases rapidly, while the mass $m_{t}$ varies more slowly and goes on increasing for some time. We can imagine that by turbulent mixing at the interface, material which has lost part of its hydrogen in the convective core is continually being drawn into more external regions. However, as the exhaustion of hydrogen in the internal region proceeds, it is seen that we reach a stage when $\mu_{i} / \mu_{e}$ is of the order of 1.5 , at which the mass $m_{t}$ reaches a maximum. At this point, the fraction of the total hydrogen content of the star which has been used is still small, of the order of 15 per cent if we start with $\mu_{i}=\mu_{e}=1$. However, since the mass of the external part $\left(M-m_{t}\right)$, where $\mu=\mu_{e}=1$, cannot increase, there are difficulties in following the evolution beyond this stage. But it should be remembered that there are many other points which remain to be investigated in this connection. In particular, for these models due to the special position of the center and the vanishing of the gravitational acceleration here, even a slight amount of dissipation may be sufficient to create a small finite zone where the exhausted hydrogen is not replenished fast enough. If that should be the case, we may expect that an isothermal core will start forming at the center even before the hydrogen is fully exhausted in the whole convective core. And, once the formation of an isothermal core has been initiated, it will grow continually at the expense of the convective region owing to the fringe in radiative equilibrium which will always surround it. We shall not, however, continue this discussion here. ${ }^{11}$

It is a pleasure to acknowledge the many interesting discussions which I had with Dr. Chandrasekhar in the course of this investigation.

${ }^{10}$ Ap. J., 100, 343, 1944.

${ }^{11}$ In this connection see M. Harrison, $A$ p. J., 105, 322, 1947, where the solutions of the "generalized Cowling model," together with solutions of models with isothermal cores, are displayed. It should, however, be remarked that the existence of the transition zone is not included in this discussion. 